\title{
Current findings of kidney biopsy including nephropathy associated with hypertension and diabetes mellitus in Korea
}

Kipyo Kim ${ }^{1,2}$, Sang Ho Lee ${ }^{3,4}$, Sung Woo Lee ${ }^{5,6}$, Jung Pyo Lee ${ }^{7,8}$, Ho Jun Chin ${ }^{2,8}$, and on behalf of the Korean GlomeruloNEphritis sTudy (KoGNET) Group

\begin{abstract}
${ }^{1}$ Department of Internal Medicine, Inha University School of Medicine, Incheon; ${ }^{2}$ Department of Internal Medicine, Seoul National University Bundang Hospital, Seongnam; ${ }^{3}$ Department of Internal Medicine, Kyung Hee University Hospital at Gangdong, Seoul; ${ }^{4}$ Department of Internal Medicine, Kyung Hee University School of Medicine, Seoul; ${ }^{5}$ Department of Internal Medicine, Nowon Eulji Medical Center, Eulji University School of Medicine, Seoul; ${ }^{6}$ Department of Internal Medicine, Eulji University School of Medicine, Daejeon; ${ }^{7}$ Department of Internal Medicine, Seoul Metropolitan Government Seoul National University Boramae Medical Center, Seoul; ${ }^{8}$ Department of Internal Medicine, Seoul National University College of Medicine, Seoul, Korea
\end{abstract}

\section{Received: May 4, 2020 Revised : June 22, 2020 Accepted: July 2, 2020}

\section{Correspondence to Ho Jun Chin, M.D. Department of Internal Medicine, Seoul National University Bundang Hospital, 82 Gumi-ro 173beon-gil, Bundang- gu, Seongnam 13620, Korea Tel: $+82-31-787-7025$ Fax: $+82-31-787-4052$ E-mail: mednep@hanmail.net https://orcid.org/0000-0002- 3710-0190}

Background/Aims: This study aimed to investigate long-term temporal trends and outcomes of biopsy-proven kidney diseases in a multicenter kidney biopsy cohort, focusing on hypertension and diabetes, the leading causes of end-stage kidney disease (ESKD).

Methods: The study included a total of 21,426 patients who underwent kidney biopsy from 1979 to 2018 in 18 hospitals in Korea. We selected subgroups of adults with diabetes $(\mathrm{n}=2,813)$ or clinically presumed hypertensive nephrosclerosis (HT$\mathrm{N}, \mathrm{n}=2,917$ ). Clinical, demographic, and laboratory data were collected in conjunction with pathologic findings. The prevalence of pathologically confirmed kidney diseases over time and their associations with clinical outcomes were evaluated.

Results: The prevalence of biopsy-proven diabetic nephropathy (DN) has increased significantly from $2.5 \%$ to $6.0 \%$ in the total cohort in the recent 30 years with an increase in the prevalence of diabetes. Approximately $68 \%$ of total diabetic patients had non-diabetic renal disease (NDRD); the proportion was retained since 2000s. DN showed a significantly higher risk of ESKD than NDRD (hazard ratio [HR], 1.59; 95\% confidence interval [CI], 1.35 to 1.88). The prevalence of biopsy-proven HT-N remained $<2 \%$ in the total cohort for several decades. There was no difference in risks of ESKD between patients with or without biopsy-proven HT-N (HR, 0.93; 95\% CI, 0.54 to 1.59).

Conclusions: In recent decades, the prevalence of diabetes and DN has significantly increased in the kidney biopsy cohort, showing an increased risk of ESKD. Despite the large numbers of patients meeting the clinical criteria of HT-N, most of those were diagnosed with pathologic diagnoses other than HT-N.

Keywords: Diabetic nephropathies; Nephrosclerosis; Glomerulonephritis; Biopsy 


\section{INTRODUCTION}

Kidney biopsy has remained a golden standard for the diagnosis of renal parenchymal diseases despite recent efforts to develop non-invasive diagnostic approaches $[1,2]$. The usefulness of kidney biopsy includes providing evidence for diagnosis, prognosis, and response to therapy of different kidney diseases [3]. In particular, kidney biopsy registries encompass many kinds of kidney diseases and can display their epidemiology and clinicopathologic associations [4]. Previous kidney biopsy cohort studies demonstrated different prevalence of several types of glomerulonephritis (GN) according to ethnicities and geographic regions [5]. Nevertheless, the majority of these studies were single-center studies with a small number of patients and a short follow-up period [6-8]. As a result, knowledge of temporal trends in the diagnosis of biopsy-proven kidney diseases is insufficient and inconsistent between studies. For several decades, there have been substantial changes in comorbidities such as diabetes, obesity, and hypertension, medications, and lifestyles, all of which could affect the prevalence of biopsy-proven kidney diseases. The global prevalence of diabetes increased dramatically from $4.3 \%$ in 1980 to $9.0 \%$ in 2014 [9], and in a systemic review, the recent prevalence of hypertension was reported as high as $26.4 \%$ worldwide [10]. Hypertension and diabetes are well known as two of the leading etiologies of end-stage kidney disease (ESKD) [11]. Although histological findings of diabetic nephropathy (DN) and hypertensive nephrosclerosis (HT-N) have been described in detail and classified in previous studies [12-14], the cause of chronic kidney disease (CKD) is clinically diagnosed without kidney biopsy in many diabetic and hypertensive patients. Furthermore, a considerable number of patients with diabetes or hypertension were found to have pathologic diagnoses other than DN or HT-N in kidney biopsy $[15,16]$. Therefore, hypertension- and diabetes-related ESKD may be overestimated, and the actual contributions of diabetes and hypertension to CKD could be biased. Although kidney biopsy has not been performed routinely to confirm HT-N or DN, kidney biopsy is now more frequently performed in diabetic and hypertensive patients. Therefore, in this study, we aimed to investigate the long-term temporal trends and outcomes of pathologically diagnosed kidney diseases in multicenter kidney biopsy registries, especially including kidney diseases related to diabetes and hypertension.

\section{METHODS}

\section{Study population and data collection}

We initially screened 21,617 patients with native kidney biopsy between 1979 and 2018 from 18 hospitals throughout Korea, retrospectively. First, in a total of 21,426 patients after excluding 191 patients with renal cancer or tumor, we investigated clinical characteristics and overall trends of biopsy-confirmed kidney diseases (Supplementary Fig. 1). Second, we selected two subgroups of adult patients with diabetes $(\mathrm{n}=2,813)$ or clinically presumed HT-N ( $\mathrm{n}=2,917)$ after excluding 976 children aged $<18$ years and 237 patients with missing age at kidney biopsy to explore the pathologic diagnoses and their associations with clinical outcomes in each group. Kidney biopsy was performed with ultrasonography-guided percutaneous biopsy, and the results were interpreted by a renal pathologist in each hospital. The clinical data at the time of biopsy and the last follow-up were queried into the hospital information system (HIS) with the primary keys of the patients' identification number and date of kidney biopsy at each hospital. All 18 centers have an HIS, and data were scanned and saved before launching the HIS. Five trained research nurses had recorded the predefined parameters on the template database file based on the queried information, and one of nephrologists of this research had cleansed the data for analysis. Data of final outcomes, incidences of ESKD and death, were gathered from each hospital's HIS, the ESKD registry of the Korean Society of Nephrology which was started in 1980, and the Statistics of Korea, and were merged based on the national identification number. The follow-up duration was $110.6 \pm 105.0$ months for ESKD and 119.4 \pm 106.6 months for death.

This study was conducted in accordance with the Declaration of Helsinki and approved by the Institutional Review Board (IRB) of Seoul National University Bundang Hospital (B-1707/408-106) and the other 17 centers. Written consent was waived by the IRB because of the retrospective nature of the study. 

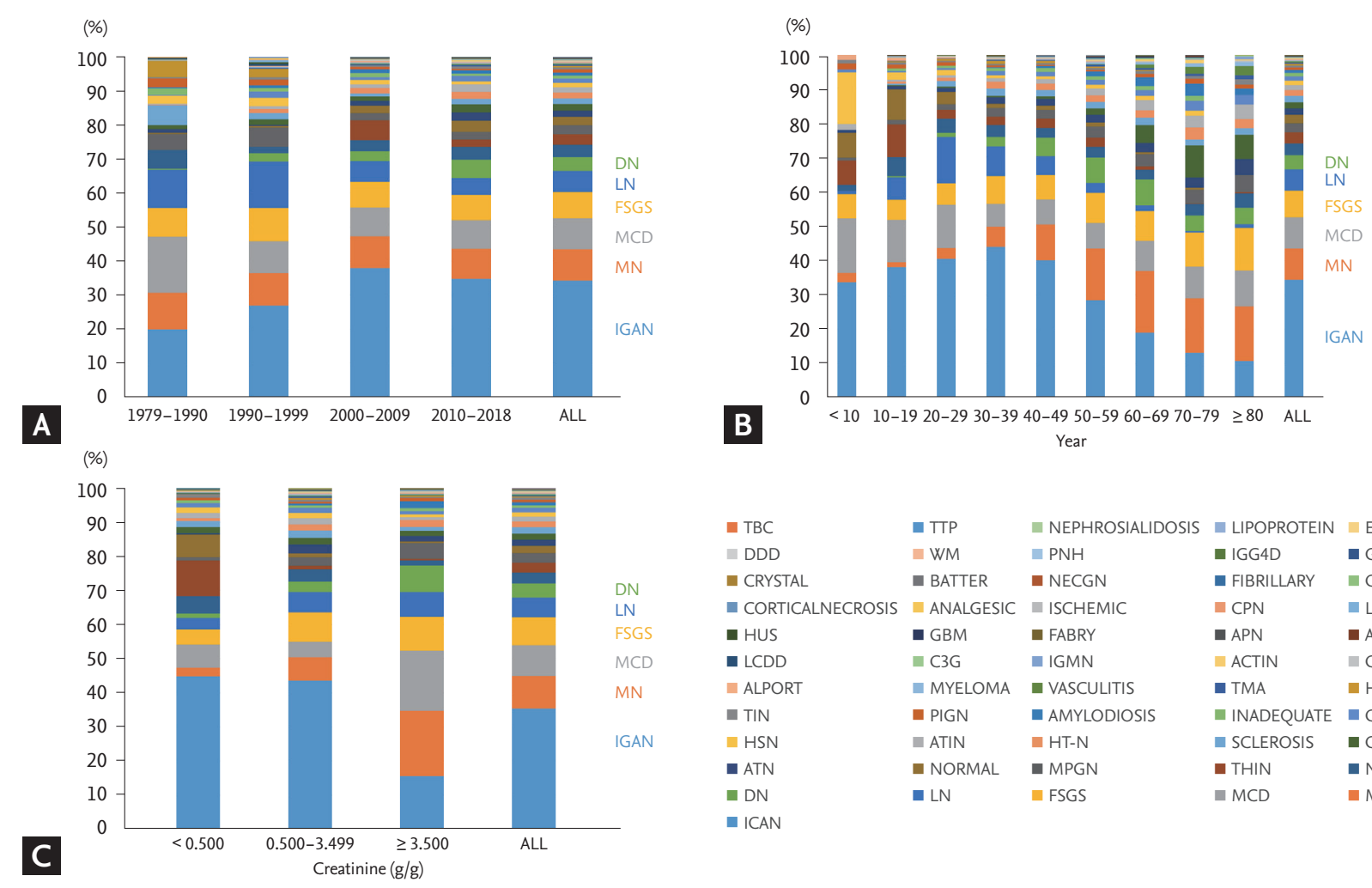

\begin{tabular}{|c|c|c|c|c|}
\hline TBC & = TTP & NEPHROSIALIDOSIS & IIPOPROTEIN & $=\mathrm{ECD}$ \\
\hline$=\mathrm{DDD}$ & WM & 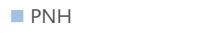 & - IGG4D & - GRANULOMA \\
\hline E CRYSTAL & - BATTER & - NECGN & — FIBRILLARY & = CRYO \\
\hline = CORTICALNECROSIS & ANALGESIC & ISCHEMIC & $=\mathrm{CPN}$ & = LCN \\
\hline - HUS & - GBM & - FABRY & - APN & - ANTIGBM \\
\hline - LCDD & C3G & IGMN & ACTIN & CIQ \\
\hline ALPORT & MYELOMA & - VASCULITIS & - TMA & - HFRS \\
\hline TIN & - PIGN & AMYLODIOSIS & INADEQUATE & $\|$ CTIN \\
\hline HSN & ATIN & $=\mathrm{HT}-\mathrm{N}$ & SCLEROSIS & CRESGN \\
\hline - ATN & - NORMAL & MPGN & - THIN & NONSPFGN \\
\hline$\square \mathrm{DN}$ & - LN & FSGS & $=\mathrm{MCD}$ & $\mathrm{MN}$ \\
\hline ICAN & & & & \\
\hline
\end{tabular}

Figure 1. Trends of pathologic diagnosis in kidney biopsy during the past 40 years in Korea. (A) Pathologic diagnosis according to periods. (B) Pathologic diagnosis according to age at kidney biopsy. (C) Pathologic diagnosis according to urine protein-to-creatinine ratio. DN, diabetic nephropathy; LN, lupus nephritis; FSGS, focal segmental glomerulosclerosis; MCD, minimal change disease; MN, membranous nephropathy; IGAN, IgA nephropathy; TBC, tuberculosis; TTP, thrombotic thrombocytopenic purpura; ECD, Erdheim-chester disease; DDD, dense deposition disease; WM, Waldenstrom's macroglobulinemia; PNH, paroxysmal nocturanl hemoglobinuria; IGG4D, IgG4 related disease; NECGN, nectrotizing glomerulonephritis; CRYO, cryoglobulinemic glomerulonephritis; CPN, chronic pyelonephritis; LCN, liver cirrhosis-related nephropathy; HUS, hemolytic uremic syndrome; GBM, glomerular basement membrane; APN, acute pyelonephritis; LCDD, light chain deposition disease; $\mathrm{C}_{3} \mathrm{G}, \mathrm{C}_{3}$ glomerulopathy; IGMN, IgM nephropathy; TMA, thrombotic microangiopathy; HFRS, hemorrahgic fever with renal syndrome; TIN, tubulointerstitial nephritis; PIGN, post-infectious glomerulonephritis; CTIN, chronic tubulointerstitial nephritis; HSN, Henoch-Schonlein nephritis; ATIN, acute tubulointerstitial nephritis; HT-N, hypertensive nephrosclerosis; CRESGN, crescentic glomerulonephritis; ATN, acute tubular necrosis; MPGN, membranoproliferative glomerulonephritis; THIN, thin membrane disease; NONSPFGN, non-specific glomerulonephritis.

\section{Definitions of covariates}

Diabetes mellitus was defined as random glucose level $\geq 200 \mathrm{mg} / \mathrm{dL}$, hemoglobin A1c $\geq 6.5 \%$, taking antidiabetic medication, or diagnosed by the physician. Hypertension was defined as systolic blood pressure (SBP) $\geq 140 \mathrm{mmHg}$, diastolic blood pressure $\geq 90 \mathrm{mmHg}$, taking antihypertensive medication to control blood pressure, or diagnosed by the physician. The estimated glomerular filtration rate (eGFR) was calculated using the original Modification of Diet in Renal Disease equation for adults and the height-independent equation for children [17]. Nephrotic syndrome was defined as urine protein-to-creatinine ratio (UPCR) $\geq 3.5 \mathrm{~g} / \mathrm{g}$ and serum albumin $<3.0 \mathrm{~g} / \mathrm{dL}$. For the comparison between clinical and pathologic entity of HT-N, we used the following clinical diagnostic criteria of HT-N: (1) presence of hypertension, (2) no diabetes, (3) no evidence of vasculitis and lupus nephritis (LN), (4) low-grade proteinuria defined as UPCR $<2.0 \mathrm{~g} / \mathrm{g}$, which was similar to those in previous studies $[16,18]$. Clinically diagnosed HT-N patients were divided into three groups: biopsy-proven HT-N without other pathologic diagnoses, pathologic diagnoses other than HT-N (non-HT-N), and biopsy-proven HT-N combined with other pathologic diagnoses (mixed). The pathologic diagnosis of diabetic patients was grouped into pure DN, non-diabetic 
Table 1. Pathologic diagnoses of 21,426 patients with native kidney biopsy.

\begin{tabular}{|c|c|c|c|}
\hline Group & Pathologic diagnosis & Number & $\%$ \\
\hline \multirow[t]{24}{*}{ Glomerulonephritis } & IgA nephropathy (IGAN) & 7,586 & 34.17 \\
\hline & Membraous nephropathy (MN) & 2,035 & 9.17 \\
\hline & Minimal change disease (MCD) & 2,028 & 9.13 \\
\hline & Focal segmental glomerulosclerosis (FSGS) & 1,698 & 7.65 \\
\hline & Lupus nephritis (LN) & 1,398 & 6.30 \\
\hline & Diabetic nephropathy (DN) & 887 & 3.99 \\
\hline & Membranoproliferative glomerulonephritis (MPGN) $)^{\mathrm{a}}$ & 585 & 2.63 \\
\hline & Crescentic glomerulonephritis (CRESGN) & 410 & 1.85 \\
\hline & Henoch-Schonlein nephritis (HSN) & 290 & 1.31 \\
\hline & Post-infectious glomerulonephritis (PIGN) ${ }^{\mathrm{b}}$ & 177 & 0.80 \\
\hline & Thrombotic microangiopathy (TMA) ${ }^{c}$ & 75 & 0.34 \\
\hline & Vasculitis & 66 & 0.30 \\
\hline & Ciq nephropathy (C1QN) & 51 & 0.23 \\
\hline & IgM nephropathy (IGMN) & 40 & 0.18 \\
\hline & $\mathrm{C}_{3}$ glomerulopathy $\left(\mathrm{C}_{3} \mathrm{G}\right)$ & 34 & 0.15 \\
\hline & Anti-glomerular basement membrane nephritis (ANTIGBM) & 19 & 0.09 \\
\hline & Hemolytic uremic syndrome (HUS) & 14 & 0.06 \\
\hline & Liver cirrhosis-related nephropathy (LCN) & 12 & 0.05 \\
\hline & Cryoglobulinemic glomerulonephritis (CRYO) & 5 & 0.02 \\
\hline & Nectrotizing glomerulonephritis (NECGN) & 3 & 0.01 \\
\hline & $\mathrm{IgG}_{4}$ related disease $\left(\mathrm{IGG}_{4} \mathrm{D}\right)$ & 2 & 0.01 \\
\hline & Paroxysmal nocturanl hemoglobinuria ( $\mathrm{PNH})$ & 2 & 0.01 \\
\hline & Dense deposition disease (DDD) & 1 & 0.00 \\
\hline & Thrombotic thrombocytopenic purpura (TTP) & 1 & 0.00 \\
\hline \multirow[t]{8}{*}{ Tubulointerstitial lesion } & Acute tubular necrosis (ATN) & 412 & 1.86 \\
\hline & Acute tubulointerstitial nephritis (ATIN) & 340 & 1.53 \\
\hline & Chronic tubulointerstitial nephritis (CTIN) & 276 & 1.24 \\
\hline & Tubulointerstitial nephritis (TIN) & 127 & 0.57 \\
\hline & Hemorrahgic fever with renal syndrome (HFRS) & 110 & 0.50 \\
\hline & Acute and chronic tubulointerstitial nephritis (ACTIN) & 50 & 0.23 \\
\hline & Batter syndrome (BATTER) & 2 & 0.01 \\
\hline & Interstitial granuloma (GRANULOMA) & 2 & 0.01 \\
\hline \multirow{3}{*}{$\begin{array}{l}\text { Glomerular basement membrane } \\
\text { abnormality (GBM) lesion }\end{array}$} & Thin membrane disease (THIN) & 690 & 3.11 \\
\hline & Alport's syndrome (ALPORT) & 58 & 0.26 \\
\hline & Non-specified glomerular basement membrane abnormality (GBM) & 14 & 0.06 \\
\hline \multirow[t]{5}{*}{ Paraproteinemia-related lesion } & Amyloidosis & 196 & 0.88 \\
\hline & Myeloma kidney (MYELOMA) & 63 & 0.28 \\
\hline & Light chain deposition disease (LCDD) & 35 & 0.16 \\
\hline & Fibrillary glomerulonephritis (FIBRILLARY) & 5 & 0.02 \\
\hline & $\begin{array}{l}\text { Waldenstrom's macroglobulinemia related proliferative } \\
\text { glomerulonephritis (WM) }\end{array}$ & 2 & 0.01 \\
\hline
\end{tabular}


Table 1. Continued

\begin{tabular}{|c|c|c|c|}
\hline Group & Pathologic diagnosis & Number & $\%$ \\
\hline \multirow[t]{3}{*}{ Ischemic lesion } & Hypertensive nephrosclerosis (HT-N) & 372 & 1.68 \\
\hline & Ischemic nephropathy (ISCHEMIC) & 7 & 0.03 \\
\hline & Cortical necrosis (CORTICALNECROSIS) & 6 & 0.03 \\
\hline \multirow[t]{9}{*}{ Miscellaneous lesion } & Acute pyelonephritis (APN) & 15 & 0.07 \\
\hline & Fabry's disease (FABRY) & 15 & 0.07 \\
\hline & Chronic pyelonephritis (CPN) & 11 & 0.05 \\
\hline & Analgesic nephropathy (ANALGESIC) & 6 & 0.03 \\
\hline & Crystal nephropathy (CRYSTAL) & 2 & 0.01 \\
\hline & Tuberculosis (TBC) & 1 & 0.00 \\
\hline & Erdheim-chester disease (ECD) & 1 & 0.00 \\
\hline & Lipoprotein nephropathy (LIPOPROTEIN) & 1 & 0.00 \\
\hline & Nephrosialidosis & 1 & 0.00 \\
\hline \multirow[t]{4}{*}{ Not specified lesion } & No abnormality (NORMAL) & 546 & 2.46 \\
\hline & Diffuse global sclerosis (SCLEROSIS) ${ }^{\mathrm{d}}$ & 393 & 1.77 \\
\hline & $\begin{array}{l}\text { Non-specific glomerulonephritis without mesangial proliferative } \\
\text { lesion(NONSPFGN_MESE) }\end{array}$ & 550 & 2.48 \\
\hline & $\begin{array}{l}\text { Non-specific glomerulonephritis with mesangial proliferative lesion } \\
(\text { NONSPFGN_MESP })^{f}\end{array}$ & 260 & 1.17 \\
\hline Inadequate sample & Inadequate sample (INADEQUATE)g & 215 & 0.97 \\
\hline Sum & & 22,203 & 100.00 \\
\hline
\end{tabular}

There were 777 patients with two main diagnoses and, therefore, 22,203 diagnoses in all patients.

${ }^{a}$ Original diagnosis of MPGN included $18 \mathrm{C}_{3} \mathrm{G}$ in the second-look decision at this research.

${ }^{\mathrm{b}} \mathrm{PIGN}$ includes post-streptococcal glomerulonephritis.

${ }^{\circ}$ TMA does not include cases of TTP or HUS.

${ }^{\mathrm{d}}$ SCLEROSIS was defined with global sclerosis more than $50 \%$ which could not be categorized in a specific diagnosis.

${ }^{\mathrm{e}}$ NONSPFGN_MESP showed mesangial proliferation without definite diagnosis, such as IGAN.

${ }^{\mathrm{f}}$ NONSPFGN_MESE showed mesangial expansion without mesangial proliferation which could not be categorized into a definite diagnosis.

gINADEQUATE had the information of no appropriate tissue samples for light microscopic examination, immunofluorescent microscopic examination, and electron microscopic examination in the original pathologic reports.

renal disease (NDRD), and NDRD superimposed on DN (NDRD/DN).

\section{Pathologic diagnosis}

We primarily adopted the findings and diagnosis by the pathologist in each hospital. We enrolled all pathologic diagnoses to describe the overall trends based on the results of renal biopsies, including data of light microscopic examination, immunofluorescent microscopic examination, and electron microscopic examination. Among 21,426 patients, 777 have two pathologic diagnoses; therefore, we analyzed a total of 22,203 diagno- ses. All pathologic diagnoses are shown in Table 1. We defined primary GN as IgA nephropathy (IGAN), minimal change disease (MCD), membranous nephropathy (MN), focal segmental glomerulosclerosis (FSGS), membranoproliferative glomerulonephritis (MPGN), and $\mathrm{C}_{3}$ glomerulopathy $\left(\mathrm{C}_{3} \mathrm{G}\right)$ regardless of the pathogenesis.

\section{Statistical analysis}

All analyses were performed using IBM SPSS Statistics version 25.o (IBM Co., Armonk, NY, USA) and R software version 3.6.1 (R Foundation for Statistical Computing, Vienna, Austria). Descriptive statistics were reported as 
Table 2. Temporal trends in patient characteristics and the incidence of kidney biopsy

\begin{tabular}{|c|c|c|c|c|c|c|}
\hline Period of biopsy & $\begin{array}{c}\text { Total }^{\mathrm{a}} \\
(\mathrm{n}=21,426)\end{array}$ & $\begin{array}{c}1979-1989 \\
(n=1,598)\end{array}$ & $\begin{array}{c}1990-1999 \\
(n=1,392)\end{array}$ & $\begin{array}{c}2000-2009 \\
(n=7,252)\end{array}$ & $\begin{array}{l}2010-2018 \\
(n=11,065)\end{array}$ & $p$ value \\
\hline Annual biopsy rate, p.m.p./yr & 11.59 & 3.61 & 3.11 & 15.06 & 24.26 & \\
\hline Age, yr & $42.1 \pm 17.7$ & $32.5 \pm 12.7$ & $38.7 \pm 14.7$ & $40.1 \pm 17.0$ & $45.1 \pm 18.3$ & $<0.001$ \\
\hline Children & $976(4.6)$ & $162(10.5)$ & $59(4 \cdot 3)$ & $354(4 \cdot 9)$ & $401(3.6)$ & $<0.001$ \\
\hline Male sex & $11,565(54.0)$ & $933(58.4)$ & $764(54.9)$ & $3,876(53 \cdot 5)$ & $5,909(53 \cdot 4)$ & 0.002 \\
\hline Hypertension & $10,994(53.0)$ & $837(66.9)$ & $704(52.3)$ & $3,135(43 \cdot 7)$ & $6,254(57.5)$ & $<0.001$ \\
\hline Diabetes & $2,833(14.0)$ & $18(2.0)$ & $78(5 \cdot 9)$ & $803(11.2)$ & $1,932(17 \cdot 9)$ & $<0.001$ \\
\hline $\mathrm{SBP}, \mathrm{mmHg}$ & $127 \cdot 3 \pm 19 \cdot 4$ & $131.1 \pm 21.8$ & $130.0 \pm 21.8$ & $125.2 \pm 18.4$ & $127.6 \pm 19.1$ & $<0.001$ \\
\hline $\mathrm{DBP}, \mathrm{mmHg}$ & $78.6 \pm 13.4$ & $88.2 \pm 16.2$ & $84.2 \pm 13.8$ & $77.7 \pm 12.2$ & $77.0 \pm 12.7$ & $<0.001$ \\
\hline Albumin, g/dL & $3.4 \pm 0.9$ & $3.0 \pm 1.0$ & $3.0 \pm 0.9$ & $3.6 \pm 0.9$ & $3.5 \pm 0.9$ & $<0.001$ \\
\hline Hemoglobin, g/dL & $12.6 \pm 2.4$ & $13.1 \pm 2.8$ & $12.3 \pm 2.7$ & $12.8 \pm 2.3$ & $12.5 \pm 2.3$ & $<0.001$ \\
\hline eGFR, $\mathrm{mL} / \mathrm{min} / 1.73 \mathrm{~m}^{2}$ & $77.1 \pm 60.4$ & $62.3 \pm 33.2$ & $66.9 \pm 37.8$ & $80.0 \pm 82.8$ & $78.3 \pm 45.9$ & $<0.001$ \\
\hline$\geq 90$ & $7,664(37 \cdot 1)$ & $243(17.6)$ & $357(26.8)$ & $2,745(38.7)$ & $4,319(39 \cdot 9)$ & \\
\hline $60-89$ & $5,553(26.9)$ & $510(36.9)$ & $429(32.2)$ & $2,095(29.5)$ & $2,519(23.2)$ & \\
\hline $45-59$ & $2,294(11.1)$ & $231(16.7)$ & $156(11.7)$ & $75^{8}(10.7)$ & $1,149(10.6)$ & \\
\hline $30-44$ & $1,952(9 \cdot 5)$ & $150(10.8)$ & $135(10.1)$ & $592(8.3)$ & $1075(9.9)$ & \\
\hline $15^{-29}$ & $1,690(8.2)$ & $110(8.0)$ & $124(9 \cdot 3)$ & $475(6.7)$ & $981(9.1)$ & \\
\hline$<15$ & $1,492(7.2)$ & $139(10.1)$ & $130(9.8)$ & $429(6.0)$ & $794(7 \cdot 3)$ & \\
\hline UPCR, g/g Cr & $3 \cdot 3 \pm 4.2$ & $3.9 \pm 4.6$ & $4 \cdot 5 \pm 4 \cdot 7$ & $3.0 \pm 3.9$ & $3 \cdot 3 \pm 4.1$ & $<0.001$ \\
\hline Neprotic syndrome & $3,420(17.5)$ & $377(27 \cdot 3)$ & $423(32.5)$ & $845(13.2)$ & $1750(16.9)$ & $<0.001$ \\
\hline
\end{tabular}

Values are presented as mean \pm SD or number (\%).

p.m.p., per million population; SBP, systolic blood pressure; DBP, diastolic blood pressure; eGFR, estimated glomerular rate; UPCR, urine protein-creatinine ratio.

${ }^{a} 219$ patients with missing data on the date of renal biopsy were included.

mean \pm standard deviation for continuous variables or frequency for categorical variables. Differences in continuous variables were analyzed by $t$ test and one-way analysis of variance (ANOVA) test and, in categorical variables, by chi-square test. Multiple comparisons were performed using Tukey-Kramer post hoc test. The independent risk factors to estimate the final outcomes were assessed by Cox hazard proportional model adjusted for related variables. The proportion of missingness of variables is shown in Supplementary Table 1, and multiple imputation by chained equation with classifications and regression trees was used for handling missing data. Complete case analyses were also performed as sensitivity analyses. Clinical characteristics and outcomes of patients with biopsy-proven HT-N were compared with those of patients without HT-N using propensity score matching for related covariates. The standardized mean difference was estimated to examine the balance of covariate distribution between the matched cohorts. Two-sided $p$ values were reported with 0.05 taken as the level of statistical significance.

\section{RESULTS}

Overall trends of pathologic diagnosis during 40 years Overall temporal trends in clinical characteristics of the enrolled participants are shown in Table 2. Among 21,426 patients, 11,565 (54.0\%) were men, 976 (4.6\%) were children aged $<18$ years, and the mean age was $42.1 \pm 17.7$ years. There were 10,659 (53.0\%) hypertensive patients and 2,813 (14.0\%) diabetic patients. Nearly one-fourth of patients had eGFR $<45 \mathrm{~mL} / \mathrm{min} / 1.73 \mathrm{~m}^{2}$ at the time of biopsy. The average incidence of kidney 

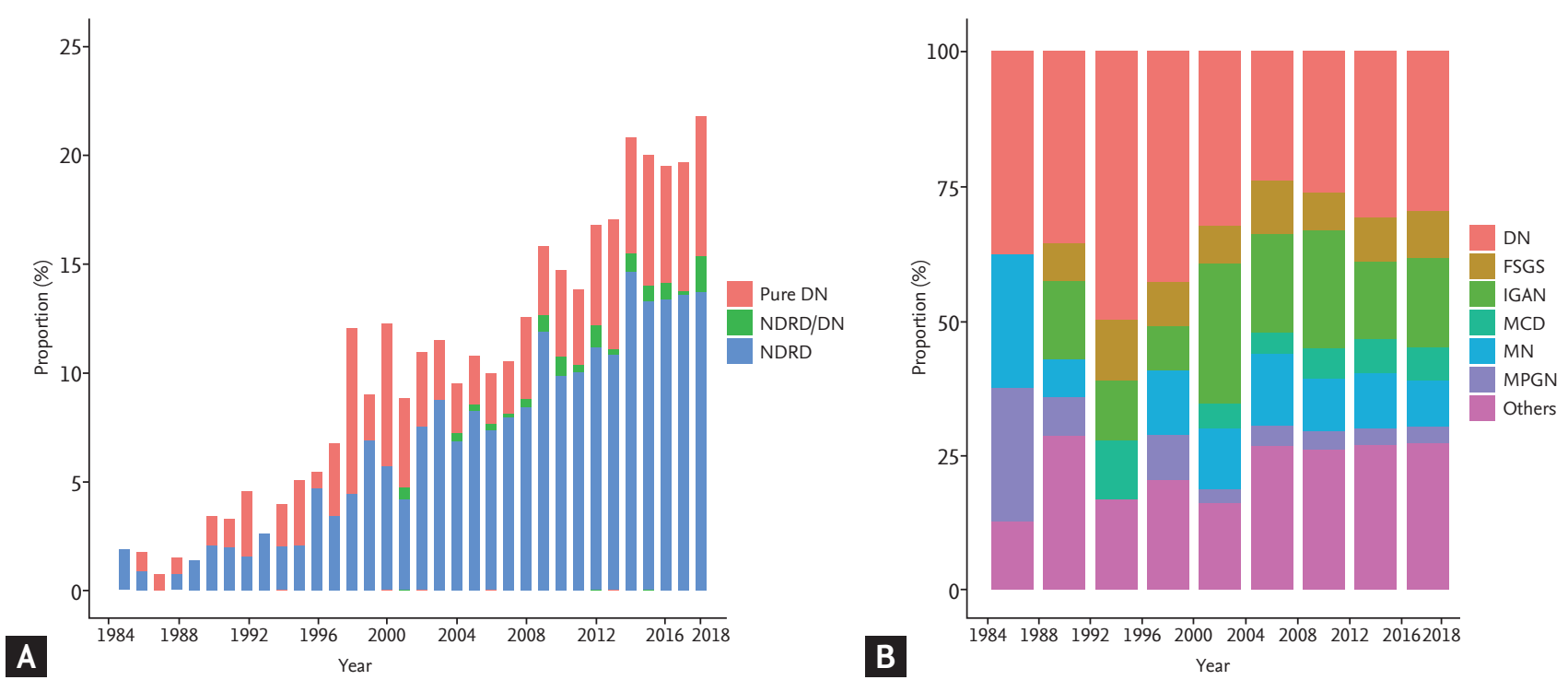

Figure 2. Temporal trends of renal pathologic diagnosis in diabetic patients. (A) Annual proportions of pure diabetic nephropathy (DN), non-diabetic renal disease (NDRD)/DN, NDRD in the total study cohort. (B) Annual proportions of DN (pure DN plus NDRD/DN) and other pathologic diagnoses in total diabetic patients. FSGS, focal segmental glomerulosclerosis; IGAN, IgA nephropathy; MCD, minimal change disease; MN, membranous nephropathy; MPGN, membranoproliferative glomerulonephritis.

biopsy during 40 years was 11.59 per million population/year. The most frequent pathologic diagnosis was IGAN (34.17\%, $\mathrm{n}=7,586)$, followed by MN (9.17\%, $\mathrm{n}=$ 2,035), MCD (9.13\%, $\mathrm{n}=2,028)$, FSGS (7.65\%, $\mathrm{n}=1,698)$, LN $(6.30 \%, n=1,398), \mathrm{DN}(3.99 \%, \mathrm{n}=887)$, and MPGN and $\mathrm{C}_{3} \mathrm{G}(2.79 \%, \mathrm{n}=619)$; therefore, the prevalence of primary GN was $62.90 \%$. Over the follow-up period, the frequency of biopsy in older patients gradually increased, and the frequency of biopsy in diabetic patients increased rapidly from $2.0 \%$ in 1979 to 1989 to $17.9 \%$ in 2010 to 2018 . The mean eGFR of the participants was higher after 2000 than that in the previous study period. Nephrotic syndrome was found in approximately $17.5 \%$ of patients with kidney biopsy. Proteinuria levels and the proportion of nephrotic syndrome at the time of biopsy decreased after 2000 .

The prevalence of IGAN increased from 19.9\% (1979 to 1989 ) to $38.0 \%$ (2000 to 2009) and then stabilized at approximately $35 \%$ (Fig. 1A). The prevalence of MCD, MN, and FSGS barely changed since 1990. However, the prevalence of MPGN decreased from $4.7 \%$ to $5.7 \%$ (1979 to 1999) to $2.3 \%$ (2010 to 2018). The prevalence of LN also decreased from $13.6 \%$ (1990 to 1999) to $4.9 \%$ (2010 to 2018). IGAN was the most frequent GN in all age groups, except in patients aged $\geq 70$ years, among whom MN was the most frequent GN (Fig. 1B). MCD was the second most frequent pathologic diagnosis in patients aged $<20$ years and showed a second peak of incidence in patients in their sixties. The frequency of MN was increased in patients aged $>40$ years and was the most frequent diagnosis in patients aged $\geq 70$ years. Crescentic GN was among the top five GN in patients aged $\geq 70$ years. The most frequent primary GN with nephrotic range proteinuria was MN (19.2\%), followed by $\operatorname{MCD}(17.6 \%)$, IGAN (15.6\%), FSGS (9.9\%), and DN (7.8\%) (Fig. 1C).

\section{Pathology and prognosis of kidney diseases in dia- betic patients}

Among 2,813 diabetic patients, DN and NDRD were present in 881 (31.3\%) and 1,932 (68.7\%) patients, respectively. Among DN patients, pure DN was detected in $790(28.1 \%)$ patients and NDRD/DN was found in 91 (3.2\%) patients. The most common NDRD was IGAN (23.1\%), followed by MN (14.5\%), FSGS (11.0\%), MCD (7.5\%), and MPGN (4.2\%) (Supplementary Table 2). Frequencies of MPGN and FSGS were relatively higher in NDRD patients than in non-diabetic patients considering age at kidney biopsy. In patients with NDRD/DN, renal pathologies other than DN were IGAN (37.4\%), 
Table 3. Clinical characteristics of diabetic patients with kidney biopsy

\begin{tabular}{|c|c|c|c|c|}
\hline Characteristic & NDRD & NDRD/DN & Pure DN & $p$ value \\
\hline Number & 1,932 & 91 & 790 & \\
\hline Age, yr & $57 \cdot 4 \pm 14.2$ & $49.7 \pm 16.7$ & $52.9 \pm 12.7$ & $<0.001^{\mathrm{a}, \mathrm{b}}$ \\
\hline Male sex & $1,120(58.0)$ & $51(56.0)$ & $509(64.4)$ & $0.01^{\mathrm{a}}$ \\
\hline Hypertension & $1,563(81.0)$ & $73(80.2)$ & $676(86.0)$ & $0.01^{\mathrm{a}}$ \\
\hline Period of biopsy & & & & $0.01^{\mathrm{a}}$ \\
\hline $1979-1989$ & $13(0.7)$ & - & $5(0.6)$ & \\
\hline $1990-1999$ & $43(2.2)$ & - & $34(4 \cdot 3)$ & \\
\hline $2000-2009$ & $575(29.8)$ & $22(24.2)$ & $200(25 \cdot 3)$ & \\
\hline $2010-2018$ & $1,301(67.3)$ & $69(75.8)$ & $551(69.7)$ & \\
\hline $\mathrm{SBP}, \mathrm{mmHg}$ & $130.1 \pm 20.5$ & $133.7 \pm 20.7$ & $140.5 \pm 23.0$ & $<0.001^{\mathrm{a}, \mathrm{c}}$ \\
\hline $\mathrm{DBP}, \mathrm{mmHg}$ & $77.5 \pm 12.5$ & $78.8 \pm 12.3$ & $81.2 \pm 12.9$ & $<0.001^{\mathrm{a}}$ \\
\hline eGFR, $\mathrm{mL} / \mathrm{min} / 1.73 \mathrm{~m}^{2}$ & $57 \cdot 9 \pm 51.3$ & $63.2 \pm 41.8$ & $47.1 \pm 38.7$ & $<0.001^{\mathrm{a}, \mathrm{c}}$ \\
\hline Albumin, g/dL & $3.2 \pm 0.9$ & $3.3 \pm 0.7$ & $3.1 \pm 0.7$ & $<0.001^{\mathrm{a}, \mathrm{c}}$ \\
\hline Hemoglobin, g/dL & $11.8 \pm 2.4$ & $11.9 \pm 2.5$ & $10.7 \pm 2.2$ & $<0.001^{\mathrm{a}, \mathrm{c}}$ \\
\hline UPCR, g/g Cr & $4.6 \pm 4.9$ & $4.1 \pm 4.7$ & $6.1 \pm 5.1$ & $<0.001^{\mathrm{a}, \mathrm{c}}$ \\
\hline
\end{tabular}

Values are presented as mean \pm SD or number (\%). Adjusted $p$ values for multiple comparisons were obtained using the Tukey-Kramer method.

NDRD, non-diabetic renal disease; DN, diabetic nephropathy; SBP, systolic blood pressure; DBP, diastolic blood pressure; eGFR, estimated glomerular filtration rate; UPCR, urine protein-to-creatinine ratio.

${ }^{a}$ Adjusted $p<0.001$ pure DN vs. NDRD.

${ }^{\mathrm{b}}$ Adjusted $p<0.001 \mathrm{NDRD} / \mathrm{DN}$ vs. NDRD.

${ }^{\mathrm{c}}$ Adjusted $p<0.05$ pure DN vs. NDRD/DN.

Table 4. Associations between biopsy-proven diagnosis and clinical outcomes in diabetic patients

\begin{tabular}{lcccc}
\hline Outcome & B & HR & 95\% CI for HR & p value \\
\hline ESKD & & & & \\
Pure DN & 0.47 & 1.59 & $1.35-1.88$ & $<0.001$ \\
NDRD/DN & 0.11 & 1.12 & $0.70-1.78$ & 0.64 \\
NDRD & & & Reference & \\
Death & & & & \\
Pure DN & -0.19 & 0.83 & $0.58-1.18$ & 0.30 \\
NDRD/DN & 0.10 & 1.11 & $0.49-2.53$ & 0.81 \\
NDRD & & & Reference & \\
\hline
\end{tabular}

HRs were adjusted for age, sex, period of kidney biopsy, presence of hypertension, estimated glomerular filtration rate, serum albumin, hemoglobin, urine protein-to-creatinine ratio, systolic blood pressure, and diastolic blood pressure.

$\mathrm{B}$, beta coefficients; HR, hazard ratio; CI, confidence interval; ESKD, end-stage kidney disease; DN, diabetic nephropathy; NDRD, non-diabetic renal disease.

acute tubular necrosis (8.8\%), FSGS (8.8\%), tubuloint- erstitial nephritis (5.5\%), and MPGN (5.5\%). Since 1990s, as the number of diabetic patients who underwent kidney biopsy continuously increased, the proportion of biopsy-proven $\mathrm{DN}$ also increased by over 2 -fold, i.e., from $2.5 \%$ to $3.0 \%$ (1990 to 2009 ) to $6.0 \%$ (2015 to 2018 ) (Fig. 2A). The proportion of patients with DN among those with diabetes who underwent kidney biopsy was higher in 1990 s than in 2000 s (44.8\% vs. $26.3 \%, p$ $=0.001$ by chi-square test) (Fig. $2 \mathrm{~B}$ ), but no significant change has been observed since 2000. The prevalence of NDRD has been more than double that of DN since 2000s. The clinical characteristics of diabetic patients according to the presence of DN are shown in Table 3. Pure DN patients showed higher blood pressure and more severe renal impairment and proteinuria. Pure DN was associated with 1.59-fold higher risks (95\% confidence interval [CI], 1.35 to 1.88) of incident ESKD than NDRD $(p<0.001)$ (Table 4$)$, which was consistent with the results of the complete case analyses (Supplementary Table 3). However, the risk of mortality was not 
Table 5. Clinical characteristics of patients with clinical HT-N

\begin{tabular}{|c|c|c|c|c|}
\hline Characteristic & Biopsy-proven HT-N & Mixed $^{\mathrm{a}}$ & Non-HT-N ${ }^{\mathrm{b}}$ & $p$ value \\
\hline Number & 103 & 39 & 2,785 & \\
\hline Age, yr & $44.7 \pm 13.7$ & $42.9 \pm 19 \cdot 3$ & $43.6 \pm 15.1$ & 0.54 \\
\hline Male sex & $70(68.0)$ & $24(61.5)$ & $1,599(57.4)$ & 0.09 \\
\hline Period of biopsy & & & & $0.01^{c}$ \\
\hline $1979-1989$ & - & - & $230(8.3)$ & \\
\hline $1990-1999$ & $8(7.8)$ & - & $139(5.0)$ & \\
\hline $2000-2009$ & $22(21.4)$ & $12(30.8)$ & $708(25.4)$ & \\
\hline $2010-2018$ & $73(70.9)$ & $27(69.2)$ & $1,708(61.3)$ & \\
\hline $\mathrm{SBP}, \mathrm{mmHg}$ & $136.6 \pm 21.1$ & $132.8 \pm 21.9$ & $134.0 \pm 18.3$ & 0.24 \\
\hline $\mathrm{DBP}, \mathrm{mmHg}$ & $84.2 \pm 15.1$ & $81.4 \pm 14.1$ & $83.7 \pm 13 \cdot 3$ & 0.95 \\
\hline Pulse pressure, mmHg & $52.4 \pm 13.8$ & $51.4 \pm 12.8$ & $50.3 \pm 13.5$ & 0.12 \\
\hline $\mathrm{eGFR}, \mathrm{mL} / \mathrm{min} / 1.73 \mathrm{~m}^{2}$ & $43 \cdot 2 \pm 35 \cdot 4$ & $81.2 \pm 55.8$ & $73.7 \pm 37.2$ & $<0.001^{\mathrm{d}, \mathrm{e}}$ \\
\hline Albumin, g/dL & $3.9 \pm 0.5$ & $4.0 \pm 0.5$ & $3.9 \pm 0.6$ & 0.35 \\
\hline Hemoglobin, g/dL & $12.5 \pm 1.9$ & $13.1 \pm 2.5$ & $13.1 \pm 2.2$ & $0.01^{\mathrm{c}}$ \\
\hline UPCR, g/g Cr & $0.8 \pm 0.6$ & $0.7 \pm 0.5$ & $0.9 \pm 0.5$ & 0.37 \\
\hline
\end{tabular}

Values are presented as mean \pm SD or number (\%). The biopsy-proven HT-N group represents patients with only HT-N as pathologic diagnosis. Adjusted p values for multiple comparisons were obtained using the Tukey-Kramer method.

HT-N, hypertensive nephrosclerosis; SBP, systolic blood pressure; DBP, diastolic blood pressure; eGFR, estimated glomerular filtration rate; UPCR, urine protein-to-creatinine ratio.

${ }^{a}$ HT-N combined with other pathologic diagnoses.

${ }^{\mathrm{b}}$ Other pathologic diagnoses other than HT-N.

${ }^{\mathrm{c}}$ Adjusted $p<0.05$ HT-N vs. non-HT-N.

${ }^{\mathrm{d}}$ Adjusted $p<0.001$ HT-N vs. non-HT-N.

${ }^{\mathrm{e}}$ Adjusted $p<0.001$ HT-N vs. mixed.

Table 6. Associations between pathologic diagnoses and ESKD development in patients with clinical HT-N

\begin{tabular}{|c|c|c|c|c|}
\hline \multirow[t]{2}{*}{ Pathologic diagnosis } & \multicolumn{2}{|c|}{$\begin{array}{l}\text { Total patients with clinical HT-N } \\
\qquad(\mathrm{n}=2,927)^{\mathrm{a}}\end{array}$} & \multicolumn{2}{|c|}{$\begin{array}{c}\text { Matched cohort with biopsy-proven HT-N or } \\
\text { non-HT-N }(\mathrm{n}=103 / 206)\end{array}$} \\
\hline & $\operatorname{HR}(95 \% \mathrm{CI})$ & $p$ value & $\mathrm{HR}(95 \% \mathrm{CI})$ & $p$ value \\
\hline Biopsy-proven HT-N & $0.93(0.54-1.59)$ & 0.78 & $0.92(0.49-1.71)$ & 0.78 \\
\hline Non-HT-N & 1.0 (reference) & - & 1.0 (reference) & - \\
\hline
\end{tabular}

HRs were adjusted with age, sex, period of kidney biopsy, estimated glomerular filtration rate, serum albumin, hemoglobin, urine protein-to-creatinine ratio, systolic blood pressure, and diastolic blood pressure. The biopsy-proven HT-N group represents patients with only HT-N as pathologic diagnosis.

ESKD, end-stage kidney disease; HT-N, hypertensive nephrosclerosis; HR, hazard ratio; CI, confidence interval.

${ }^{\text {aT } T h e ~ H R ~ f o r ~ t h e ~ m i x e d ~ g r o u p ~ c o u l d ~ n o t ~ b e ~ e s t i m a t e d ~ d u e ~ t o ~ n o ~ e v e n t s . ~}$

different between pure DN and NDRD. Other risk factors of incident ESKD in diabetic patients were older age, male sex, hypertension, SBP, eGFR, serum albumin, and hemoglobin (Supplementary Table 4). The appropriate level of SBP was $<130 \mathrm{mmHg}$ on the day after admission for kidney biopsy.

\section{Pathology and prognosis of kidney diseases in patients with clinical HT-N}

First, we examined the correlation between HT-N diagnosed by clinical criteria and biopsy-proven HT-N (Supplementary Table 5). All patients with biopsy-proven HT-N had hypertension, but only $5 \%$ of the patients who 

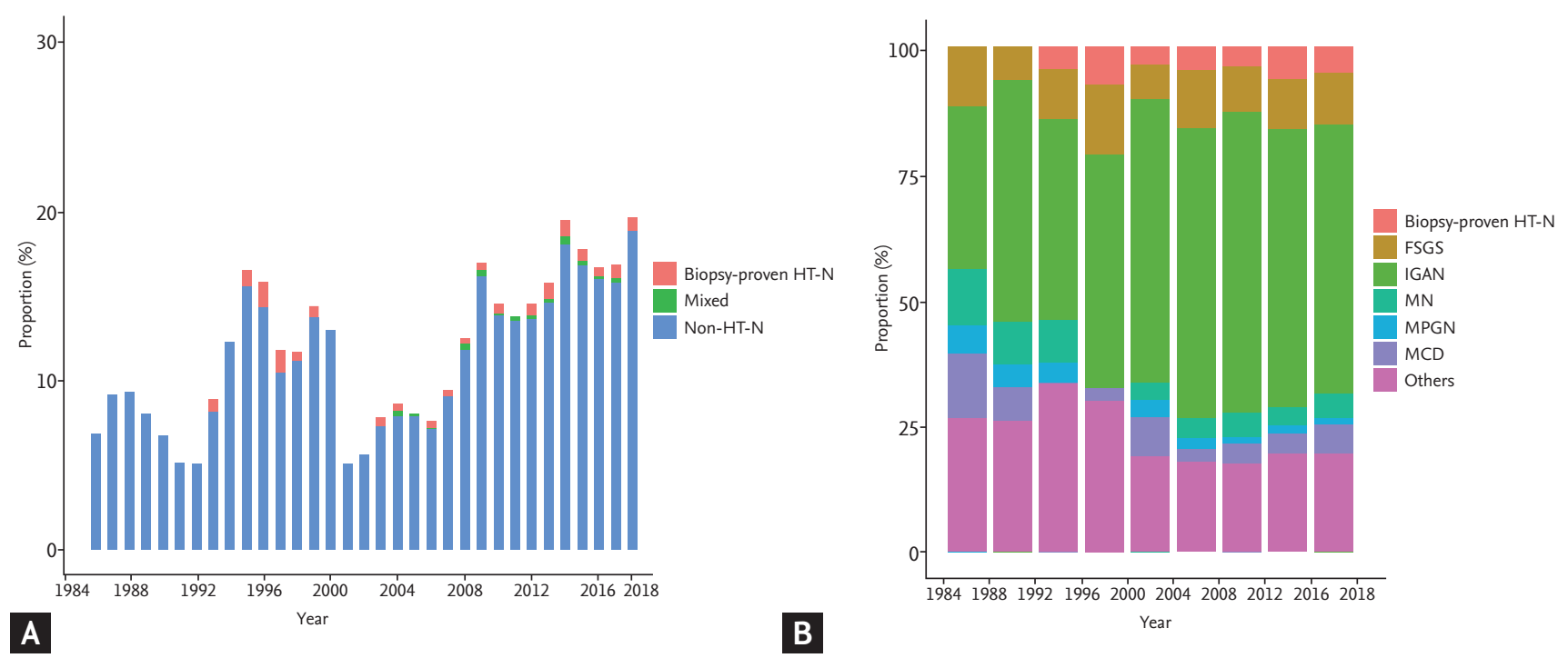

Figure 3. Temporal trends of renal pathologic diagnosis in patients with clinical hypertensive nephrosclerosis (HT-N). (A) Annual proportions of biopsy-proven HT-N (with no other pathologic diagnosis), mixed (HT-N combined with other pathologic diagnoses), and non-HT-N (pathologic diagnoses other than HT-N) in the total study cohort. (B) Annual proportions of biopsy-proven HT-N and other pathologic diagnoses in patients with clinical HT-N. FSGS, focal segmental glomerulosclerosis; IGAN, IgA nephropathy; MN, membranous nephropathy; MPGN, membranoproliferative glomerulonephritis; MCD, minimal change disease.

met the clinical criteria including low-grade proteinuria (criteria 3) were pathologically diagnosed with HT-N. Moreover, most of the biopsy-proven HT-N patients did not meet the clinical criteria; sensitivities were as low as $39 \%$. In a total of 2,927 adult patients who met the clinical criteria, there were 142 subjects with biopsy-proven HT-N and 2,785 with non-HT-N (Supplementary Table 6). Of the 142 patients with biopsy-proven HT-N, 103 (72.5\%) had only biopsy-proven HT-N with no other pathologic diagnosis. The most common biopsy-proven kidney disease in non-HT-N patients was IGAN (54.4\%), followed by FSGS (10.0\%), MN (5.0\%), and MCD (5.0\%); IGAN and FSGS were found more frequently in clinical HT-N patients than in the total cohort.

The proportion of clinical HT-N has ranged from $5 \%$ to $20 \%$ of the total cohort during the study period and showed an increasing trend after the 2000 s with an increase in an annual biopsy rate (Fig. 3A). Biopsy-proven HT-N has been observed since 1990s when the prevalence of hypertension in Korea increased obviously than before. Nevertheless, the prevalence of biopsy-proven HT-N remained quite low (approximately $5 \%$ of clinical HT-N patients and less than $2 \%$ of the total cohort) until the recent years. A majority of clinical HT-N patients were diagnosed with non-HT-N based on the kidney biopsy. Of these patients, distributions of specific kidney diseases over time were similar to those in the total cohort; an increase in IGAN and a decrease in MPGN incidence were found (Fig. 3B).

Table 5 shows the characteristics of the pathologic subgroups in clinical HT-N patients. Patients with biopsy-proven HT-N showed significantly lower eGFR and hemoglobin levels compared with non-HT-N patients. In a multivariable Cox proportional hazards model, the risk of ESKD development was not different between biopsy-proven HT-N and non-HT-N (hazard ratio [HR], $0.93 ; 95 \%$ CI, 0.54 to $1.59 ; p=0.78$ ) (Table 6), which was similar to the results of the complete case analysis (Supplementary Table 7). The risk factors of ESKD in clinical HT-N patients included eGFR, hemoglobin, and UPCR (Supplementary Table 8). For further assessment of outcomes of HT-N, we matched 103 patients with biopsy-proven HT-N and 206 non-HT-N patients using 1:2 propensity score matching for all relevant covariates (Supplementary Table 9). The standardized mean difference was less than 0.1 for all covariates after matching 
(Supplementary Table 10). The multivariable-adjusted Cox analysis in the matched group was consistent with the results before matching (HR, 0.92; 95\% CI, 0.49 to $1.71 ; p=0.78)$. The risk of death could not be assessed due to the small number of observed events in HT-N patients.

\section{DISCUSSION}

To our knowledge, this is the first multicenter-based study of kidney biopsy in Korea, which included a large number of participants with long-term follow-up. Our findings revealed the spectrum and temporal trends over 40 years in biopsy-proven DN and hypertension-related kidney disease, as well as primary or secondary GN. The incidence of kidney biopsy in diabetic patients has increased substantially in the recent 30 years, and the incidence of DN has also increased. The ratio of the number of patients with biopsy-proven DN and NDRD did not change over the recent two decades. DN showed significantly higher risk for incident ESKD than NDRD. Biopsy-proven HT-N accounted for only a small proportion of clinical HT-N patients, and since the 1990s, its prevalence has not changed significantly. No significant difference was found in the risks of ESKD between biopsy-proven HT-N and non-HT-N. For other GNs, the prevalence of IGAN gradually increased, while those of LN and MPGN decreased in the past decades.

To date, there have been several studies of kidney biopsy registry in various regions, population, and institutions. Nevertheless, these studies have mostly described only the prevalence of biopsy-proven primary or secondary GNs during the follow-up period ranging from 3 to 20 years $[6,19-23]$. In the late twentieth and early twenty-first centuries, globally, lifestyle and socioeconomic status have changed substantially, accompanying the change in the prevalence of chronic diseases such as hypertension and diabetes. Particularly, the number of diabetic patients worldwide has nearly quadrupled between 1980 and 2014 [9]. Similar epidemiology of diabetes has been reported in the Korean population [24]. In a systematic analysis for the Global Burden of Disease Study 2013, the age-standardized rates for CKD due to diabetes increased globally by $10.6 \%$ between 1990 and 2013 [25]. Our findings suggest that the increasing inci- dence of diabetes has a significant effect on kidney diseases and outcome. From 1990 to 2018, the proportion of DN in total biopsy-proven kidney diseases increased over by two-fold. Moreover, the risk for incident ESKD in DN was significantly higher than in NDRD, which was consistent with other studies $[26,27]$. Indeed, in Korea, the most common cause of ESKD was GN in the early 1990 s, but diabetes has been the most common cause since 1993 [28]. According to a nationwide survey by the Korean Society of Nephrology in 2014, 48\% of ESKD was attributable to diabetes [28], which is similar in different countries [29,30]. Since the pathologic classification of DN was proposed in 2010, there has been a growing interest in the role of kidney biopsy in diabetic patients [12]. However, the reported prevalence of DN in kidney biopsy studies was similar or lower than that of NDRD; in studies including more than 100 diabetic patients, the proportion of DN ranges from $35 \%$ to $40 \%$ and NDRD from $40 \%$ to $50 \%[5,31]$. In part, this high prevalence of NDRD could be attributed to the indication of kidney biopsy in diabetic patients. Nevertheless, in a cohort of diabetic patients with albuminuria, more than $20 \%$ of patients had NDRD confirmed by kidney biopsy [32,33]. Many diabetic patients are referred to the nephrologists at a late stage of CKD and clinically diagnosed with DN without kidney biopsy. Therefore, a large number of advanced CKD cases in diabetic patients may be caused by NDRD, and it may be an overestimate that $50 \%$ of new ESKD results from DN.

In this study, the proportion of NDRD in diabetic patients was relatively higher (68\%) and temporally unchanged since the 2000s. This indicates that, for diabetic patients, renal biopsies have been performed very selectively, and the biopsy pattern and indication have not changed for recent decades in Korea. Patients with NDRD could benefit from specific therapeutic interventions for kidney diseases [26,34]. However, currently, there is no effective clinical tool for identifying NDRD without biopsy. Kidney biopsy is mainly performed in patients who have clinical features different from those of DN described in the Kidney Disease Outcomes Quality Initiative (KDOQI) guideline [35], such as nephrotic syndrome, proteinuria without diabetic retinopathy, and short duration of diabetes. Several studies have examined the predictive factors for NDRD [15,36] and proposed diagnostic models to distinguish between DN 
and NDRD [37,38], but no significant improvement has yet been made. In this regard, further studies are needed to reduce the gap between clinical and pathologic diagnoses and to increase timely and appropriate kidney biopsy in diabetic patients.

On the other hand, the prevalence of hypertension in Korea increased from $19.8 \%$ in 1990 to approximately 28\% since 1998 [39]. Similarly, the proportion of hypertension as an underlying cause of ESKD in Korea has increased from less than $10 \%$ in 1980 os to $16.0 \%$ in 1992 and then remained at $16.0 \%$ to $21.4 \%$ [28]. Hypertension has been the second most common cause of ESKD since 2000. In our study, although biopsy-proven HT-N was found more frequently since the 1990 s than before, the proportion remained less than $2 \%$ of the entire biopsy cohort. Likewise, in other biopsy studies, the prevalence of HT-N was also as low as $2.5 \%$ to $9 \%$ [40-42]. In clinical practice, HT-N is usually diagnosed without kidney biopsy in non-diabetic patients with reduced GFR, hypertension, and low level or absence of proteinuria [43]. Kidney biopsy in hypertensive patients is selectively performed in cases such as those with increasing proteinuria, hematuria, and azotemia. Nevertheless, as shown in our results, the number of biopsy-proven HT-N is considered too small. Only $5 \%$ of clinically diagnosed HT-N patients had biopsy-proven HT-N. Similarly, in a study of 47 patients who met the clinical criteria of HT-N and underwent kidney biopsy, many cases had possible immune-complex-mediated GNs other than HT-N [44]. Actually, HT-N has been criticized as a vague umbrella term that does not correlate well with pathological findings such as arteriolar hyalinization and sclerosis [45]. Our findings indicate that the clinical criteria of HT-N are not adequate for providing pathologic diagnosis. Therefore, a substantial number of ESKD attributed to hypertension may be accompanied by other kidney diseases such as chronic GN. In addition, biopsy-proven HT-N showed no difference in risks for incident ESKD compared with non-HT-N. To date, studies of the prognosis in biopsy-proven HT-N are still limited. Recently, Ovrehus et al. [46] revealed that risks of ESKD and mortality for biopsy-proven HT-N did not differ from those for GNs, but significantly lower than those for DN. These findings suggest that the overall renal outcome of HT-N was not as poor as expected from the data in ESKD registries.
Among primary or secondary GNs, the most common GN in Korea has been IGAN, followed by MN, MCD, FSGS, and LN. This is similar to other populations in Asia such as Japan and China $[47,48]$. The prevalence of IGAN increased from 1990 to 2009 and then stabilized, and the prevalence of MPGN gradually decreased during the past decades, as shown in other single-center studies in Korea [8,19]. A decrease in MPGN may be caused by decreased infection and improvement in sanitation and socioeconomic status [49]. In addition, our findings showed a decrease in the prevalence of LN, which may result from early and effective therapeutic intervention, which hindered the development of LN [50].

In the present study, we explored the current and past spectrum and temporal trends of kidney diseases, focusing on hypertension and diabetes, the leading causes of CKD at the present era. To date, many studies have reported that the major cause of ESKD has changed from GN to diabetes and hypertension in the past decades. Although pathologic findings of DN and HT-N were found more commonly than decades ago, our findings indicate that a relatively large proportion of cases in patients with diabetes or hypertension might be attributed to other kidney diseases such as GN. This supports the need to consider kidney biopsy more actively for proper diagnostic approaches in patients with diabetes or clinical HT-N. Fortunately, kidney biopsy has increasingly been performed in patients with low-grade proteinuria or normal renal function; thus, histological diagnosis can be made earlier than before. Kidney biopsy for patients with clinically presumed DN or HT-N could provide early accurate diagnosis and appropriate management.

This study has limitations. First, despite the multicenter design, only the Korean population is included. Generalization to other populations is difficult. Ethnic factors are important in the prognosis of HT-N [51], but predispositions such as African American with apolipoprotein $\mathrm{L} 1$ variants could not be assess in this study. Second, since our study is based on retrospective data from the kidney biopsy registry, clinical variables such as laboratory tests and medications are limited. In particular, some important variables, such as duration of diabetes, presence of diabetic retinopathy, and hematuria were not available. Third, it may be unclear whether hypertension at the time of biopsy precedes kidney disease in 
hypertensive patients. However, in a real clinical setting, many hypertensive patients are referred for suspicion of kidney diseases without long-term follow-up.

In conclusion, our multicenter cohort study of kidney biopsy over 40 years showed that the incidence of DN has been rapidly increasing during the recent 30 years, contributing to ESKD development. However, NDRD was also consistently present in a majority of diabetic patients with kidney biopsy. Biopsy-proven HT-N was present in only a few of clinical HT-N patients, and the incidence remained unchanged since the $1990 \mathrm{Os}$. DN was associated with a higher risk of ESKD than NDRD, but biopsy-proven HT-N did not differ from non-HT-N in the risk of ESKD. Considering the high prevalence of NDRD or nonHT-N, further investigations are needed to determine the role of kidney biopsy in these populations.

\section{KEY MESSAGE}

1. In the recent 30 years in Korea, kidney biopsy has been increasingly performed in diabetic patients and biopsy-proven diabetic nephropathy have been found more frequently, which was associated with a higher risk of end-stage kidney disease (ESKD).

2. Biopsy-proven hypertensive nephrosclerosis (HT-N) has been found in a small proportion of patients with clinically presumed HT-N and did not differ from non-HT-N in the risk of ESKD.

3. Considering the high prevalence of non-diabetic renal disease or non-HT-N, further investigations for the role and indication of kidney biopsy might be needed.

\section{Conflict of interest}

No potential conflict of interest relevant to this article was reported.

\section{REFERENCES}

1. Connor KL, Denby L. MicroRNAs as non-invasive biomarkers of renal disease. Nephrol Dial Transplant 2019 Sep 20 [Epub]. https://doi.org/10.1093/ndt/gfz183.
2. Taherkhani A, Farrokhi Yekta R, Mohseni M, Saidijam M, Arefi Oskouie A. Chronic kidney disease: a review of proteomic and metabolomic approaches to membranous glomerulonephritis, focal segmental glomerulosclerosis, and IgA nephropathy biomarkers. Proteome Sci 2019;17:7.

3. Dhaun N, Bellamy CO, Cattran DC, Kluth DC. Utility of renal biopsy in the clinical management of renal disease. Kidney Int 2014;85:1039-1048.

4. Brkovic V, Milinkovic M, Kravljaca M, et al. Does the pathohistological pattern of renal biopsy change during time? Pathol Res Pract 2018;214:1632-1637.

5. Fiorentino M, Bolignano D, Tesar V, et al. Renal biopsy in 2015: from epidemiology to evidence-based indications. Am J Nephrol 2016;43:1-19.

6. Chiu HF, Chen HC, Lu KC, Shu KH; Taiwan Society of Nephrology. Distribution of glomerular diseases in Taiwan: preliminary report of National Renal Biopsy Registry-publication on behalf of Taiwan Society of Nephrology. BMC Nephrol 2018;19:6.

7. Al Turk AA, Estiverne C, Agrawal PR, Michaud JM. Trends and outcomes of the use of percutaneous native kidney biopsy in the United States: 5-year data analysis of the Nationwide Inpatient Sample. Clin Kidney J 2018;11:330-336.

8. Yim T, Kim SU, Park S, et al. Patterns in renal diseases diagnosed by kidney biopsy: a single-center experience. Kidney Res Clin Pract 2020;39:60-69.

9. NCD Risk Factor Collaboration (NCD-RisC). Worldwide trends in diabetes since 1980: a pooled analysis of 751 population-based studies with 4.4 million participants. Lancet 2016;387:1513-1530.

10. Kearney PM, Whelton M, Reynolds K, Muntner P, Whelton PK, He J. Global burden of hypertension: analysis of worldwide data. Lancet 2005;365:217-223.

11. Ghaderian SB, Beladi-Mousavi SS. The role of diabetes mellitus and hypertension in chronic kidney disease. J Renal Inj Prev 2014;3:109-110.

12. Tervaert TW, Mooyaart AL, Amann K, et al. Pathologic classification of diabetic nephropathy. J Am Soc Nephrol 2010;21:556-563.

13. Freedman BI, Iskander SS, Buckalew VM Jr, Burkart JM, Appel RG. Renal biopsy findings in presumed hypertensive nephrosclerosis. Am J Nephrol 1994;14:90-94.

14. Harvey JM, Howie AJ, Lee SJ, et al. Renal biopsy findings in hypertensive patients with proteinuria. Lancet 1992;340:1435-1436.

15. Fiorentino M, Bolignano D, Tesar V, et al. Renal biopsy in 
patients with diabetes: a pooled meta-analysis of 48 studies. Nephrol Dial Transplant 2017;32:97-110.

16. Zarif L, Covic A, Iyengar S, Sehgal AR, Sedor JR, Schelling JR. Inaccuracy of clinical phenotyping parameters for hypertensive nephrosclerosis. Nephrol Dial Transplant 2000;15:1801-1807.

17. Levey AS, Bosch JP, Lewis JB, Greene T, Rogers N, Roth D. A more accurate method to estimate glomerular filtration rate from serum creatinine: a new prediction equation. Modification of Diet in Renal Disease Study Group. Ann Intern Med 1999;130:461-470.

18. Fogo A, Breyer JA, Smith MC, et al. Accuracy of the diagnosis of hypertensive nephrosclerosis in African Americans: a report from the African American Study of Kidney Disease (AASK) Trial. AASK Pilot Study Investigators. Kidney Int 1997;51:244-252.

19. Shin HS, Cho DH, Kang SK, et al. Patterns of renal disease in South Korea: a 20-year review of a single-center renal biopsy database. Ren Fail 2017;39:540-546.

20. Su S, Yu J, Wang Y, Wang Y, Li J, Xu Z. Clinicopathologic correlations of renal biopsy findings from northeast China: a 10-year retrospective study. Medicine (Baltimore) 2019;98:e15880.

21. Zhou Q, Yang X, Wang M, et al. Changes in the diagnosis of glomerular diseases in east China: a 15-year renal biopsy study. Ren Fail 2018;40:657-664.

22. Rivera F, Lopez-Gomez JM, Perez-Garcia R; Spanish Registry of Glomerulonephritis. Clinicopathologic correlations of renal pathology in Spain. Kidney Int 2004;66:898904.

23. van Paassen $P$, van Breda Vriesman PJ, van Rie $H$, Tervaert JW. Signs and symptoms of thin basement membrane nephropathy: a prospective regional study on primary glomerular disease: The Limburg Renal Registry. Kidney Int 2004;66:909-913.

24. Noh J. The diabetes epidemic in Korea. Endocrinol Metab (Seoul) 2016;31:349-353.

25. Global Burden of Disease Study 2013 Collaborators. Global, regional, and national incidence, prevalence, and years lived with disability for 301 acute and chronic diseases and injuries in 188 countries, 1990-2013: a systematic analysis for the Global Burden of Disease Study 2013. Lancet 2015;386:743-800.

26. Byun JM, Lee CH, Lee SR, et al. Renal outcomes and clinical course of nondiabetic renal diseases in patients with type 2 diabetes. Korean J Intern Med 2013;28:565-572.
27. Soleymanian T, Hamid G, Arefi M, et al. Non-diabetic renal disease with or without diabetic nephropathy in type 2 diabetes: clinical predictors and outcome. Ren Fail 2015;37:572-575.

28. Jin DC, Yun SR, Lee SW, et al. Lessons from 30 years' data of Korean end-stage renal disease registry, 1985-2015. Kidney Res Clin Pract 2015;34:132-139.

29. National Institutes of Health. 2007 Annual Data Report. Atlas of Chronic Kidney Disease and End-Stage Renal Disease in the United States. Philadelphia (PA): Saunders, 2007.

30. Atkins RC. The epidemiology of chronic kidney disease. Kidney Int Suppl 2005;94:S14-S18.

31. Bermejo S, Pascual J, Soler MJ. The current role of renal biopsy in diabetic patients. Minerva Med 2018;109:116125 .

32. Christensen PK, Larsen S, Horn T, Olsen S, Parving HH. Causes of albuminuria in patients with type 2 diabetes without diabetic retinopathy. Kidney Int 2000;58:17191731.

33. Parving HH, Gall MA, Skott P, et al. Prevalence and causes of albuminuria in non-insulin-dependent diabetic patients. Kidney Int 1992;41:758-762.

34. Li L, Zhang X, Li Z, et al. Renal pathological implications in type 2 diabetes mellitus patients with renal involvement. J Diabetes Complications 2017;31:114-121.

35. KDOQI. KDOQI clinical practice guidelines and clinical practice recommendations for diabetes and chronic kidney disease. Am J Kidney Dis 2007;49(2 Suppl 2):S12-S154.

36. Liang S, Zhang XG, Cai GY, et al. Identifying parameters to distinguish non-diabetic renal diseases from diabetic nephropathy in patients with type 2 diabetes mellitus: a meta-analysis. PLoS One 2013;8:e64184.

37. Zhou J, Chen X, Xie Y, Li J, Yamanaka N, Tong X. A differential diagnostic model of diabetic nephropathy and non-diabetic renal diseases. Nephrol Dial Transplant 2008;23:1940-1945.

38. Liu MY, Chen XM, Sun XF, et al. Validation of a differential diagnostic model of diabetic nephropathy and non-diabetic renal diseases and the establishment of a new diagnostic model. J Diabetes 2014;6:519-526.

39. Kim TJ, Lee JW, Kang HT, et al. Trends in blood pressure and prevalence of hypertension in Korean adults based on the 1998-2014 KNHANES. Yonsei Med J 2018;59:356365.

40. Takebayashi S, Kiyoshi Y, Hisano S, et al. Benign ne- 
phrosclerosis: incidence, morphology and prognosis. Clin Nephrol 2001;55:349-356.

41. Innes A, Johnston PA, Morgan AG, Davison AM, Burden RP. Clinical features of benign hypertensive nephrosclerosis at time of renal biopsy. Q J Med 1993;86:271-275.

42. Vikse BE, Aasarod K, Bostad L, Iversen BM. Clinical prognostic factors in biopsy-proven benign nephrosclerosis. Nephrol Dial Transplant 2003;18:517-523.

43. Freedman BI, Cohen AH. Hypertension-attributed nephropathy: what's in a name? Nat Rev Nephrol 2016;12:2736.

44. Wang XC, Liu CH, Chen YJ, et al. Clinical and pathological analysis of the kidney in patients with hypertensive nephropathy. Exp Ther Med 2013;6:1243-1246.

45. Meyrier A. Nephrosclerosis: a term in quest of a disease. Nephron 2015;129:276-282.

46. Ovrehus MA, Oldereid TS, Dadfar A, et al. Clinical phenotypes and long-term prognosis in white patients with biopsy-verified hypertensive nephrosclerosis. Kidney Int Rep 2019;5:339-347.
47. Sugiyama H, Yokoyama H, Sato H, et al. Japan Renal Biopsy Registry and Japan Kidney Disease Registry: committee report for 2009 and 2010. Clin Exp Nephrol 2013;17:155-173.

48. Li LS, Liu ZH. Epidemiologic data of renal diseases from a single unit in China: analysis based on 13,519 renal biopsies. Kidney Int 2004;66:920-923.

49. McGrogan A, Franssen CF, de Vries CS. The incidence of primary glomerulonephritis worldwide: a systematic review of the literature. Nephrol Dial Transplant 2011;26:414-430.

50. Moroni G, Vercelloni PG, Quaglini S, et al. Changing patterns in clinical-histological presentation and renal outcome over the last five decades in a cohort of 499 patients with lupus nephritis. Ann Rheum Dis 2018;77:1318-1325.

51. Kopp JB. Rethinking hypertensive kidney disease: arterionephrosclerosis as a genetic, metabolic, and inflammatory disorder. Curr Opin Nephrol Hypertens 2013;22:266272. 
KJIM

Supplementary Table 1. The proportion of missingness of data

\begin{tabular}{|c|c|c|c|c|}
\hline \multirow[b]{2}{*}{ Variable } & \multicolumn{4}{|c|}{ Missingness of data } \\
\hline & $\begin{array}{l}\text { Total cohort } \\
(\mathrm{n}=21,426)\end{array}$ & $\begin{array}{l}\text { Diabetic patients } \\
\qquad(\mathrm{n}=2,813)\end{array}$ & $\begin{array}{c}\text { Patients with } \\
\text { clinical HT-N }(n=2,927)\end{array}$ & $\begin{array}{c}\text { Matched cohort with biopsy-proven } \\
\text { HT-N or non-HT-N }(\mathrm{n}=309)\end{array}$ \\
\hline Age, yr & $230(1.1)$ & 0 & $\mathrm{O}$ & 0 \\
\hline Sex & $4(0.02)$ & 0 & 0 & 0 \\
\hline Hypertension & $700(3 \cdot 3)$ & $7(0.2)$ & o & $\mathrm{O}$ \\
\hline Diabetes & $1,215(5 \cdot 7)$ & 0 & o & o \\
\hline Period of biopsy & $119(0.6)$ & o & $\mathrm{O}$ & o \\
\hline $\mathrm{SBP}, \mathrm{mmHg}$ & $2,301(10.7)$ & $161(5 \cdot 7)$ & $130(4.4)$ & $13(4.2)$ \\
\hline $\mathrm{DBP}, \mathrm{mmHg}$ & $2,291(10.7)$ & $158(5.6)$ & $128(4.4)$ & $12(3.9)$ \\
\hline $\mathrm{eGFR}, \mathrm{mL} / \mathrm{min} / 1.73 \mathrm{~m}^{2}$ & $781(3.6)$ & $31(1.1)$ & $35(1.2)$ & $10(3.2)$ \\
\hline Albumin, g/dL & $978(4.6)$ & $46(1.6)$ & $53(1.8)$ & $7(2.3)$ \\
\hline Hemoglobin, g/dL & $1,656(7.7)$ & $59(2.1)$ & $172(5 \cdot 9)$ & $4(1.3)$ \\
\hline UPCR, g/g Cr & $4,722(22.0)$ & $530(18.8)$ & $\mathrm{O}$ & 0 \\
\hline
\end{tabular}

Values are presented as number (\%).

HT-N, hypertensive nephrosclerosis; SBP, systolic blood pressure; DBP, diastolic blood pressure; eGFR, estimated glomerular filtration rate; UPCR, urine protein-creatinine ratio. 
Supplementary Table 2. Pathologic diagnosis in 1,932 NDRD patients

\begin{tabular}{|c|c|c|}
\hline Pathologic diagnosis & Number & $\%$ among NDRD \\
\hline IGAN & 473 & 23.1 \\
\hline $\mathrm{MN}$ & 296 & 14.5 \\
\hline FSGS & 226 & 11.0 \\
\hline MCD & 153 & 7.5 \\
\hline MPGN & 86 & 4.2 \\
\hline NONSPFGN & 85 & 4.2 \\
\hline CRESGN & 82 & 4.0 \\
\hline ATIN & 81 & 4.0 \\
\hline SCLEROSIS & 80 & 3.9 \\
\hline ATN & 76 & 3.7 \\
\hline HT-N & 73 & 3.6 \\
\hline CTIN & 47 & 2.3 \\
\hline $\mathrm{LN}$ & 42 & 2.1 \\
\hline INADEQUATE & 28 & 1.4 \\
\hline AMYLODIOSIS & 22 & 1.1 \\
\hline PIGN & 22 & 1.1 \\
\hline $\mathrm{HSN}$ & 19 & 0.9 \\
\hline NORMAL & 18 & 0.9 \\
\hline THIN & 18 & 0.9 \\
\hline TIN & 18 & 0.9 \\
\hline TMA & 18 & 0.9 \\
\hline VASCULITIS & 18 & 0.9 \\
\hline ACTIN & 17 & 0.8 \\
\hline MYELOMA & 16 & 0.8 \\
\hline LCDD & 5 & 0.2 \\
\hline $\mathrm{C}_{3} \mathrm{G}$ & 4 & 0.2 \\
\hline IGMN & 4 & 0.2 \\
\hline ANTIGBM & 3 & 0.1 \\
\hline $\mathrm{C} 1 \mathrm{Q}$ & 3 & 0.1 \\
\hline $\mathrm{LC}-\mathrm{N}$ & 3 & 0.1 \\
\hline APN & 2 & 0.1 \\
\hline FIBRILLARY & 2 & 0.1 \\
\hline ALPORT & 1 & 0.0 \\
\hline ANALGESIC & 1 & 0.0 \\
\hline CPN & 1 & 0.0 \\
\hline CRYO & 1 & 0.0 \\
\hline ECD & 1 & 0.0 \\
\hline ISCHEMIC & 1 & 0.0 \\
\hline HFRS & 1 & 0.0 \\
\hline Number of diagnosis & 2,047 & \\
\hline Number of patients & 1,932 & \\
\hline Number of patients with two diagnosis & 115 & \\
\hline
\end{tabular}

NDRD, non-diabetic renal disease; IGAN, IgA nephropathy; MN, membranous nephropathy; FSGS, focal segmental glomerulosclerosis; MCD, minimal change disease; MPGN, membranoproliferative glomerulonephritis; NONSPFGN, non-specific glomerulonephritis; CRESGN, crescentic glomerulonephritis; ATIN, acute tubulointerstitial nephritis; ATN, acute tubular necrosis; HT-N, hypertensive nephrosclerosis; CTIN, chronic tubulointerstitial nephritis; LN, lupus nephritis; PIGN, post-infectious glomerulonephritis; HSN, Henoch-Schonlein nephritis; THIN, thin membrane disease; TIN, tubulointerstitial nephritis; TMA, thrombotic microangiopathy; ACTIN, acute and chronic tubulointerstitial nephritis; LCDD, light chain deposition disease; $\mathrm{C}_{3} \mathrm{G}$, $\mathrm{C}_{3}$ glomerulopathy; IGMN, IgM nephropathy; GBM, glomerular basement membrane; LC-N, liver cirrhosis-related nephropathy; APN, acute pyelonephritis; CPN, chronic pyelonephritis; CRYO, cryoglobulinemic glomerulonephritis; ECD, Erdheim-chester disease; HFRS, hemorrahgic fever with renal syndrome. 
Supplementary Table 3. Associations between biopsy-proven diagnosis and clinical outcomes in complete cases of diabetic patients $(n=2,078)$

\begin{tabular}{|c|c|c|c|c|}
\hline Outcomes & B & $\mathrm{HR}$ & $95 \%$ CI for HR & $p$ value \\
\hline \multicolumn{5}{|l|}{ Death } \\
\hline Pure DN & -0.25 & 0.78 & $0.52-1.15$ & 0.21 \\
\hline NDRD/DN & -0.13 & 0.88 & $0.32-2.41$ & 0.81 \\
\hline NDRD & & & Reference & \\
\hline \multicolumn{5}{|l|}{ ESKD } \\
\hline Pure DN & 0.55 & 1.74 & $1.44-2.10$ & $<0.001$ \\
\hline NDRD/DN & 0.01 & 1.01 & $0.58-1.76$ & 0.98 \\
\hline NDRD & & & Reference & \\
\hline
\end{tabular}

HRs were adjusted for age, gender, period of renal biopsy, presence of hypertension, estimated glomerular filtration rate, serum albumin, hemoglobin, urine protein-to-creatinine ratio, systolic blood pressure, and diastolic blood pressure.

B, beta coefficients; HR, hazard ratio; CI, confidence interval; DN, diabetic nephropathy; NDRD, non-diabetic renal disease; ESKD, end-stage kidney disease. 
Supplementary Table 4 . Risk factors of ESKD in adult diabetic patients.

\begin{tabular}{|c|c|c|c|c|}
\hline Variable & B & HR & $95 \% \mathrm{CI}$ & $p$ value \\
\hline Male sex & 0.21 & 1.23 & $1.02-1.48$ & 0.03 \\
\hline Age, yr & -0.02 & 0.98 & $0.98-0.99$ & $<0.001$ \\
\hline Hypertension (presence) & 0.37 & 1.45 & $1.08-1.96$ & 0.01 \\
\hline \multicolumn{5}{|c|}{ SBP (compared to $120-129 \mathrm{mmHg}$ ) } \\
\hline$<110$ & 0.13 & 1.14 & $0.75^{-1.72}$ & 0.55 \\
\hline $110-119$ & 0.09 & 1.10 & $0.78-1.55$ & 0.61 \\
\hline $130-139$ & 0.52 & 1.68 & $1.23-2.30$ & 0.001 \\
\hline $140-149$ & 0.30 & 1.35 & $0.99-1.87$ & 0.06 \\
\hline$\geq 150$ & 0.31 & 1.36 & $1.00-1.85$ & $<0.05$ \\
\hline $\mathrm{eGFR}, \mathrm{mL} / \mathrm{min} / 1.73 \mathrm{~m}^{2}$ & -0.03 & 0.97 & $0.97-0.98$ & $<0.001$ \\
\hline Serum albumin, g/dL & -0.21 & 0.81 & $0.70-0.93$ & 0.004 \\
\hline Hemoglobin, g/dL & -0.13 & 0.88 & $0.84-0.92$ & $<0.001$ \\
\hline \multicolumn{5}{|c|}{ Pathology (compared to NDRD) } \\
\hline NDRD/DN & 0.01 & 1.01 & $0.58-1.78$ & 0.96 \\
\hline Pure DN & 0.57 & 1.76 & $1.46-2.12$ & $<0.001$ \\
\hline
\end{tabular}

Model adjusted with age, gender, presence of hypertension, SBP, diastolic blood pressure, serum albumin, hemoglobin, glomerular filtration rate, and urine protein-to-creatinine ratio.

ESKD, end-stage kidney disease; B, beta coefficient; HR, hazard ratio; CI, confidence interval; SBP, systolic blood pressure; eGFR, estimated glomerular filtration rate; NDRD, non-diabetic renal disease; DN, diabetic nephropathy. 


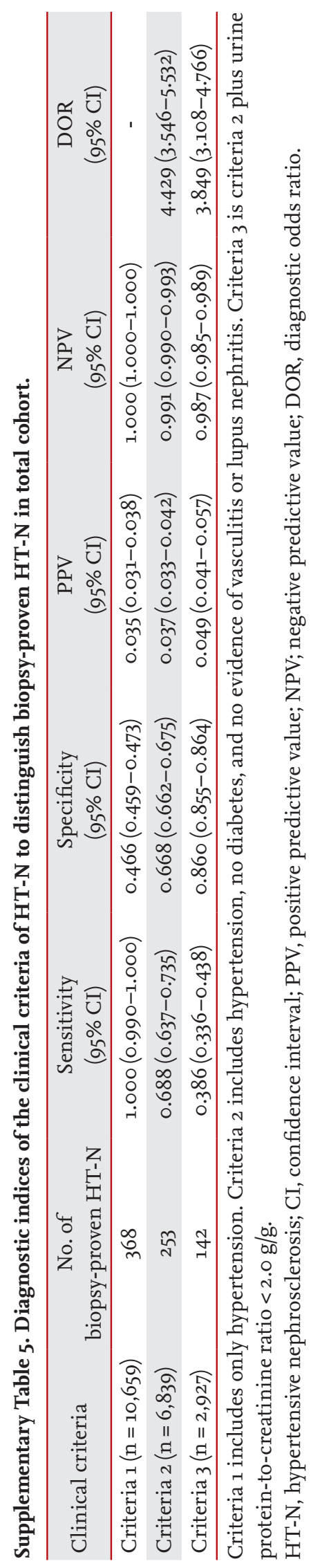


Supplementary Table 6. Pathologic diagnosis in 2,927 patients with clinical HT-N

\begin{tabular}{|c|c|c|c|}
\hline & Biopsy-proven HT-N & Non-HT-N ${ }^{\mathrm{a}}$ & Sum \\
\hline IGAN & $17(9.4)$ & $1,566(54.4)$ & $1,583(51.7)$ \\
\hline $\mathrm{MN}$ & $3(1.7)$ & $143(5.0)$ & $146(4.8)$ \\
\hline FSGS & $4(2.2)$ & $288(10.0)$ & $292(9.5)$ \\
\hline MCD & o & $143(5.0)$ & $143(4.7)$ \\
\hline $\mathrm{DN}$ & o & o & o \\
\hline $\mathrm{LN}$ & o & o & o \\
\hline MPGN & $1(5.5)$ & $59(2.0)$ & $60(2.0)$ \\
\hline HT-N & $142(78.5)$ & o & $142(4.6)$ \\
\hline NONSPFGN & $2(1.1)$ & $125(4 \cdot 3)$ & $127(4.1)$ \\
\hline SCLEROSIS & o & $65(2.3)$ & $65(2.1)$ \\
\hline CRESGN & ० & $25(0.9)$ & $25(0.8)$ \\
\hline ATN & $5(2.8)$ & $57(2.0)$ & $62(2.0)$ \\
\hline ATIN & $1(5.5)$ & $54(1.9)$ & $55(1.8)$ \\
\hline CTIN & $o(0)$ & $61(2.1)$ & $61(2.0)$ \\
\hline NORMAL & $2(1.1)$ & $52(2.1)$ & $54(2.0)$ \\
\hline THIN & o & $63(2.2)$ & $63(2.1)$ \\
\hline INADEQUATE & $1(5.5)$ & $24(0.8)$ & $25(0.8)$ \\
\hline PIGN & o & $18(0.6)$ & $18(0.6)$ \\
\hline HSN & o & $22(0.8)$ & $22(0.7)$ \\
\hline AMYLODIOSIS & $1(5.5)$ & $6(0.2)$ & $7(0.2)$ \\
\hline TIN & $1(5.5)$ & $22(0.8)$ & $23(0.8)$ \\
\hline TMA & $1(5.5)$ & $22(0.8)$ & $23(0.8)$ \\
\hline VASCULITIS & o & $5(0.2)$ & $5(0.2)$ \\
\hline HFRS & o & $6(0.2)$ & $6(0.2)$ \\
\hline MYELOMA & o & $3(0.1)$ & $3(0.1)$ \\
\hline ACTIN & o & $8(0.3)$ & $8(0.3)$ \\
\hline $\mathrm{C}_{3} \mathrm{G}$ & o & $7(0.2)$ & $7(0.2)$ \\
\hline $\mathrm{CrQ}$ & o & $9(0.3)$ & $9(0.3)$ \\
\hline LIGHT CAHIN & o & $4(0.1)$ & $4(0.1)$ \\
\hline IGMN & o & $4(0.1)$ & $4(0.1)$ \\
\hline ALPORT & o & $4(0.1)$ & $4(0.1)$ \\
\hline ANTIGBM & o & o & o \\
\hline LC-N & o & o & o \\
\hline HUS & o & $3(0.1)$ & $3(0.1)$ \\
\hline GBM & o & $4(0.1)$ & $4(0.1)$ \\
\hline $\mathrm{CPN}$ & o & $2(0.1)$ & $2(0.1)$ \\
\hline CORTICALNECROSIS & o & $2(0.1)$ & $2(0.1)$ \\
\hline ISCHEMIC NEPHROPATHY & o & o & o \\
\hline ANALGESIC & o & $2(0.1)$ & $2(0.1)$ \\
\hline APN & $\circ$ & $1(0.03)$ & $1(0.03)$ \\
\hline FABRY & o & o & o \\
\hline FIBRILLARY & o & o & o \\
\hline
\end{tabular}


KJIM'

Supplementary Table 6. Continued

\begin{tabular}{lccc}
\hline & Biopsy-proven HT-N & Non-HT-N & Sum \\
\hline CRYO & 0 & 0 & 0 \\
CRYSTAL & 0 & 0 & 0 \\
GRANULOMA & 0 & 0 & 0 \\
ECD & 0 & 0 & 0 \\
NECGN & 0 & 0 & $1(0.03)$ \\
TTP & 0 & $1(0.03)$ & $1(0.03)$ \\
WM & 0 & $1(0.03)$ & 3,062 \\
Sum of diagnosis & 181 & 2,881 & 2,927 \\
No. of patients & 142 & 2,785 & 135 \\
No. of patients with two diagnosis & 39 & 96 & 0 \\
\hline
\end{tabular}

Values are presented as number (\%).

HT-N, hypertensive nephrosclerosis; IGAN, IgA nephropathy; MN, membranous nephropathy; FSGS, focal segmental glomerulosclerosis; MCD, minimal change disease; DN, diabetic nephropathy; LN, lupus nephritis; MPGN, membranoproliferative glomerulonephritis; NONSPFGN, non-specific glomerulonephritis; CRESGN, crescentic glomerulonephritis; ATN, acute tubular necrosis; ATIN, acute tubulointerstitial nephritis; CTIN, chronic tubulointerstitial nephritis; THIN, thin membrane disease; PIGN, post-infectious glomerulonephritis; HSN, Henoch-Schonlein nephritis; TIN, tubulointerstitial nephritis; TMA, thrombotic microangiopathy; HFRS, hemorrahgic fever with renal syndrome; ACTIN, acute and chronic tubulointerstitial nephritis; $\mathrm{C}_{3} \mathrm{G}, \mathrm{C}_{3}$ glomerulopathy; IGMN, IgM nephropathy; GBM, glomerular basement membrane; LC-N, ; HUS, hemolytic uremic syndrome; GBM, non-specified glomerular basement membrane abnormality; CPN, chronic pyelonephritis; APN, acute pyelonephritis; CRYO, cryoglobulinemic glomerulonephritis; ECD, Erdheim-chester disease; NECGN, nectrotizing glomerulonephritis; TTP, thrombotic thrombocytopenic purpura; WM, Waldenstrom's macroglobulinemia.

${ }^{a}$ Pathologic diagnoses other than HT-N. 
Kim K, et al. Current findings of kidney biopsy

Supplementary Table 7. Associations between pathologic diagnoses and ESKD development in complete cases of patients with clinical HT-N

\begin{tabular}{lccccc}
\hline \multirow{2}{*}{ Pathologic diagnosis } & \multicolumn{2}{c}{$\begin{array}{c}\text { Patients with clinical HT-N }{ }^{\mathrm{a}} \\
(\mathrm{n}=2,490)\end{array}$} & & \multicolumn{2}{c}{$\begin{array}{c}\text { Matched cohort with biopsy-proven HT-N or } \\
\text { non-HT-N }(\mathrm{n}=86 / 164)\end{array}$} \\
\cline { 2 - 3 } \cline { 5 - 6 } & $\mathrm{HR}(95 \% \mathrm{CI})$ & pvalue & & $\mathrm{HR}(95 \% \mathrm{CI})$ & $p$ value \\
\hline Biopsy-proven HT-N & $0.92(0.53-1.60)$ & 0.77 & & $0.97(0.51-1.84)$ & 0.92 \\
Non-HT-N & 1.0 (reference) & - & & 1.0 (reference) & - \\
\hline
\end{tabular}

HRs were adjusted with age, gender, period of renal biopsy, glomerular filtration rate, serum albumin, hemoglobin, urine protein-creatinine ratio, systolic blood pressure, and diastolic blood pressure. The biopsy-proven HT-N group represents patients with only HT-N as pathologic diagnosis. Mixed, HT-N combined with other pathologic diagnoses; non-HT-N, other pathologic diagnoses other than HT-N.

ESKD, end-stage kidney disease; HT-N, hypertensive nephrosclerosis; HR, hazard ratio; CI, confidence interval.

${ }^{a}$ The HR for the mixed group could not be estimated due to no events. 
Supplementary Table 8. Risk factors of ESKD in patients with clinical HT-N

\begin{tabular}{lcccc}
\hline Variable & B & HR & $95 \%$ CI & p value \\
\hline GFR, mL/min/1.73 m m $^{2}$ & -0.03 & 0.97 & $0.96-0.98$ & $<0.001$ \\
Hemoglobin, g/dL & -0.07 & 0.93 & $0.87-0.99$ & 0.03 \\
UPCR, g/g Cr & 0.40 & 1.49 & $1.17-1.90$ & 0.001 \\
\hline
\end{tabular}

Model was adjusted with age, gender, and related factors to ESKD, such as presence of hypertension, diastolic blood pressure, systolic blood pressure, serum albumin, hemoglobin, GFR, and UPCR.

ESKD, end-stage kidney disease; HT-N, hypertensive nephrosclerosis; HR, hazard ratio; CI, confidence interval; GFR, glomerular filtration rate; UPCR, urine protein-to-creatinine ratio. 
Kim K, et al. Current findings of kidney biopsy

Supplementary Table 9. Clinical characteristics of matched cohort among patients with biopsy-proven HT-N or non-HT-N

\begin{tabular}{|c|c|c|c|}
\hline Characteristic & Non-HT-N & Biopsy-proven HT-N & $p$ value \\
\hline Number & 206 & 103 & \\
\hline Age, yr & $44.5 \pm 13.5$ & $44.7 \pm 13.7$ & 0.897 \\
\hline Male sex & $149(72.3)$ & $70(68.0)$ & 0.507 \\
\hline \multicolumn{4}{|l|}{ Period of biopsy } \\
\hline $1979-1989$ & - & - & \\
\hline $1990-1999$ & $15(7 \cdot 3)$ & $8(7.8)$ & \\
\hline $2000-2009$ & $46(22.3)$ & $22(21.4)$ & \\
\hline $2010-2018$ & $145(70.4)$ & $73(70.9)$ & \\
\hline $\mathrm{SBP}, \mathrm{mmHg}$ & $139 \cdot 3 \pm 23 \cdot 7$ & $137.8 \pm 23.0$ & 0.607 \\
\hline $\mathrm{DBP}, \mathrm{mmHg}$ & $86.0 \pm 16.2$ & $84.7 \pm 15.6$ & 0.482 \\
\hline $\mathrm{eGFR}, \mathrm{mL} / \mathrm{min} / 1.73 \mathrm{~m}^{2}$ & $44.9 \pm 36.5$ & $43 \cdot 3 \pm 35 \cdot 2$ & 0.711 \\
\hline Albumin, g/dL & $3.9 \pm 0.5$ & $3.9 \pm 0.5$ & 0.988 \\
\hline Hemoglobin, g/dL & $12.3 \pm 2.3$ & $12.4 \pm 1.9$ & 0.637 \\
\hline UPCR, g/g Cr & $0.8 \pm 0.5$ & $0.8 \pm 0.6$ & 0.654 \\
\hline
\end{tabular}

Values are presented as mean $\pm \mathrm{SD}$ or number (\%).

HT-N, hypertensive nephrosclerosis; SBP, systolic blood pressure; DBP, diastolic blood pressure; eGFR, estimated glomerular filtration rate; UPCR, urine protein-creatinine ratio. 
KJIM'

Supplementary Table 10. Standardized mean differences between biopsy-proven HT-N and non-HT-N before and after propensity score matching

\begin{tabular}{|c|c|c|}
\hline \multirow{2}{*}{ Variable } & \multicolumn{2}{|c|}{ Standardized mean differences } \\
\hline & Pre-matching & After-matching \\
\hline Age, yr & 0.081 & 0.016 \\
\hline Male sex & 0.106 & -0.044 \\
\hline $\mathrm{SBP}, \mathrm{mmHg}$ & 0.108 & -0.063 \\
\hline $\mathrm{DBP}, \mathrm{mmHg}$ & 0.024 & -0.087 \\
\hline \multicolumn{3}{|l|}{ Period of biopsy } \\
\hline $1979-1989$ & -0.083 & 0.000 \\
\hline $1990-1999$ & 0.028 & 0.005 \\
\hline $2000-2009$ & -0.041 & -0.010 \\
\hline $2010-2018$ & 0.096 & 0.005 \\
\hline $\mathrm{eGFR}, \mathrm{mL} / \mathrm{min} / 1.73 \mathrm{~m}^{2}$ & -0.869 & -0.046 \\
\hline Albumin, g/dL & 0.095 & 0.002 \\
\hline Hemoglobin, g/dL & -0.330 & 0.062 \\
\hline UPCR, g/g Cr & -0.042 & 0.051 \\
\hline
\end{tabular}

HT-N, hypertensive nephrosclerosis; SBP, systolic blood pressure; DBP, diastolic blood pressure; eGFR, estimated glomerular filtration rate; UPCR, urine protein-to-creatinine ratio. 


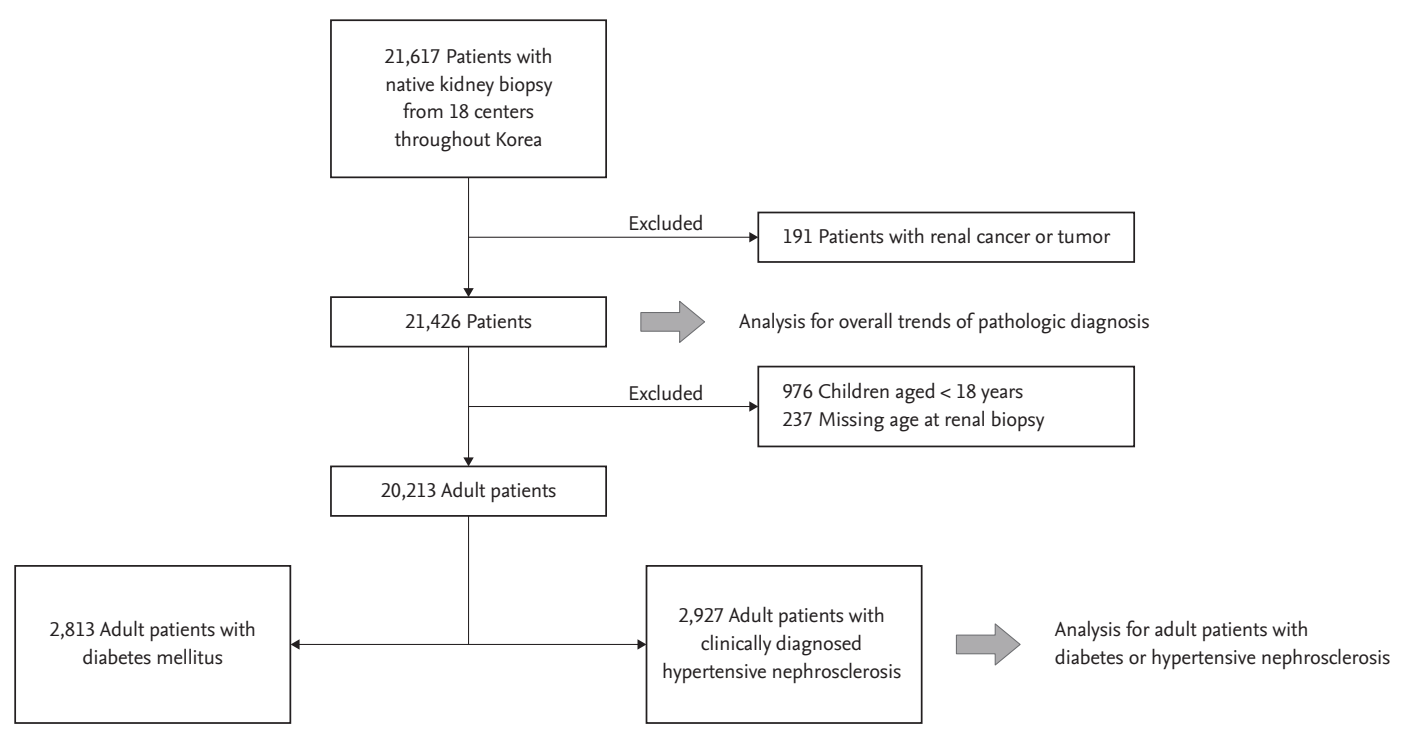

Supplementary Figure 1. Enrollment of patients. 